\title{
La Pedagogía Social ante el proceso de convergencia europea de la Educación Superior
}

\author{
Jose Antonio Caride Gómez \\ Universidad de Santiago de Compostela
}

\section{Resumen}

En los inicios del siglo XXI las universidades europeas afrontan uno de sus procesos de reforma estructural y curricular más decisivos, con objetivos y líneas de actuación que tienen como principal soporte la Declaración de Bolonia (junio de 1999). Un documento con el que se propone crear un sistema de grados académicos fácilmente comprensibles y comparables, fomentar la movilidad de los estudiantes, docentes e investigadores, garantizar la calidad de la enseñanza y crear un Espacio Europeo de la Educación Superior (EEES).

En este contexto, el artículo argumenta acerca de las connotaciones pedagógicas y sociales inherentes a estas reformas, valorando los principios y actuaciones en los que se sustentan como una nueva oportunidad para el reencuentro de las universidades consigo mismas y con la sociedad. También se analiza el estado de cuestión actual de la Pedagogía Social y de la Educación Social en España ante el proceso de convergencia, de sus realidades y desafíos en la construcción de una sociedad educadora, garante de los der e chos cívicos y la condición ciudadana. Para concluir con una serie de recomendaciones y propuestas destinadas a mejorar las decisiones que se adopten en el futuro, ante la previsible reconversión de la Diplomatura en Educación Social en un Grado con la misma denominación.

PALA B RAS CLAVE: Pedagogía Social, Educación Social, Espacio Europeo de la Educación Superior, Reformas Universitarias, Convergencia Europea.

\section{Summary}

In the beginnings of the $21^{\text {st }}$ century, European Universities confront one of the processes of structural and curricular reform more decisive, with aims and lines of action that take the Bologna Declaration as a principal support (June, 1999). A document that is designed to introduce a system of academic degrees that are easy to read and compare, to foment the mobility of students, teachers and researchers, to guarantee the quality in education and to take into account the European dimension of higher Education.

In this context, the article argues about the pedagogic and social connotations inherent in these reforms, valuing the principles and actions that sustain them, as a new opportunity for the meeting of the Universi- 
ties with himself and with the society. Also it is analyzed the condition of current question about the Social Pedagogy and about the Social Education in Spain before the process of convergence, about his realities and challenges in the construction of an educational and responsible society that guarantees the civic rights and the civil condition. To conclude with a recommendations and offers destined to improve the decisions that are adopted in the future, before the predictable restructuring of the Social Education Diplomature in a Degree with the same denomination.

KEY WORDS: Social Pedagogy, Social Education, European dimension of higher Education, University reforms, European convergence.

Impartir "enseñanzas superiores" en el seno de una comunidad de maestros y estudiantes, justificó durante siglos la misión de la universidad como una institución dedicada esencialmente a la transmisión de los saberes ya establecidos, carente como estaba de los márgenes de libertad y de los recursos que se precisaban para crear y transmitir nuevos conocimientos. $\mathrm{O}$, simplemente, de desarrollarlos anticipándose o acompañado los intensos procesos de cambio social expe rimentados por la sociedad en mucho tiempo, indiferente a la profundidad de sus mutaciones y progresos. Como ha recordado Edgar Morin $(1998,19)$, el afán conservador de las Universidades se tiñó a menudo de dogmatismo y rigidez, condenando todos los avances científicos de su época, de modo que "hasta bien entrado el siglo XVIII, la ciencia moderna se formó mayoritariamente fuera de las universidades".

De hecho, habrá que esperar hasta el siglo XIX para que en sus claustros empezase a adquirir relieve la investigación como una actividad propia y autónoma, sometiendo a reflexión crítica los conocimientos heredados y las formas de cultivarlos. Desde entonces, abrazando distintos arquetipos y modelos, las universidades no han dejado de "modernizarse", dimensionando y reformando sus estructuras organizativa y las relaciones que mantiene con las sociedades que las acogen, y a las que se brinda como un bien público cuyas responsabilidades pedagógicas, culturales y científicas deben acrecentar -directa o indirectamente- el bienestar personal y colectivo de los ciudadanos.

Se entiende, en este contexto, que la universidad no haya podido ignorar los cambios demográficos, económicos, ecológicos, culturales, políticos, tecnológicos, etc. que trajo consigo la modernidad y sus reiteradas crisis.Y que, con demasiada frecuencia, se han ido plasmando en complicaciones y a dversidades de diversa naturaleza, resuel tas con desigual fortuna por las políticas ministeriales y/o por las propias universidades. Probablemente, y sin que entremos a considerar situaciones particulares, porque-como se reconocía en el preámbulo de la Declaración Mundial sobre Educación Superior en el Siglo XXI, aprobada por los participantes en la Conferencia Mundial sobre la Educación Superior, celebrada en del 5 al 9 de octubre de 1998 en la sede de la UNES$\mathrm{CO}$ en París- es una educación que "se enfrenta en todas partes a desafíos y dificultades relativos a la financiación, la igualdad de condiciones de acceso y en el transcurso de los mismos, a una mejor capacitación del personal, la formación basada en competencias, la mejora y la conservación de la calidad de la enseñanza, la investigación y los servicios, la pertinencia de los planes de estudios, las posibilidades de empleo de los diplomados, el establecimiento de acuerdos de cooperación eficaces y la igualdad de acceso a los beneficios que reporta la cooperación internacional".

Sujeta a la expansión estructural y poblacional más importante de su historia, con 
indicadores que revelan un espectacular crecimiento de los centros universitarios en todo el mundo -ya sea por iniciativa pública o privada-, así como del número de estudiantes y profesores, las últimas décadas han sido especialmente relevantes para las universidades en diferentes geografías y sociedades. En cantidad y en calidad, como realidad o expectativa asociada a un objetivo primordial: hacer compatible su caracterización como un servicio público, abierto a toda la sociedad, con las exigencias de calidad y excelencia "que son propias de una institución dedicada al desarrollo y a la transmisión de la ciencia, la tecnología y la cultura superior" (Quintanilla, 1995: 135).

En una sociedad para la que se invoca la plena conciliación -cuando no complicidad y subordinación- entre las enseñanzas dispensadas por el sistema educativo y el mercado laboral, propiciando que el adiestramiento profesional de los egresados de aquél coincida con las demandas generadas por el empleo, se ha precipitado la necesidad de reformar universal y consensuadamente las estructuras e iniciativas de las universidades a las nuevas formas de crear y difundir el conocimiento, de repensar y mejorar los procesos de enseñanza-aprendizaje, o de inscribir sus prácticas institucionales y pedagógicas en los múltiples escenarios (locales y globales, en simultáneo) que configuran el mundo que habitamos. En todo caso, sin que al mismo tiempo que se valora el importante protagonismo que ha tenido y deberá seguir teniendo la Educación Superior y las Universidades en el desarrollo económico y social de los pueblos, se insista en denunciar el "estado de crisis" al que se encuentran sometidas desde hace décadas. En cualquier país, y prácticamente en cualquier universidad o institución cuyos cometidos y funciones sociales declaren algún tipo de responsabilidad en la formación "científica, académica y profesional" de la juventud.
De ahí, antes y ahora, el ansia y la necesidad incesante de reformarlas. Y, posiblemente, también el firme deseo de que en algún momento, aclarado el camino que deberá tomarse, tenga lugar la verdadera reforma que las Universidades y la Educación Superior precisan para ser mucho más congruentes consigo mismas y con las realidades sociales a las que deben adaptarse y, simultáneamente, transformar. Un logro que desde el cierre del pasado siglo, cuando menos en Europa y a partir del acuerdo suscrito a mediados del año 2006 por 46 países, parece inexcusablemente ligado a la implementación de la Declaración de Bolonia (formalizada el 19 de junio de 1999) y a los procesos que habilita en materia de articulación, unificación y convergencia de los sistemas de Enseñanza Superior existentes en el continente. A priori, con objetivos tan loables como son consolidar y agrandar la ciudadanía europea, promover el desarrollo cultural y social, favorecer la empleabilidad, etc.

Entre el escepticismo y la resistencia de algunos, la indiferencia o el desconocimiento de un número importante de potenciales afectados (estudiantes, profesores, personal de administración y servicios, etc.) y la confianza que parecen depositar en dicho proceso por quienes están llamados a promoverlo y animarlo (Ministerios de Educación, Consejos de Dirección, Coordinación y Gestión de las Universidades, autoridades académicas, etc.), Bolonia nombra una nueva oportunidad para la reforma de las Universidades y de la formación que han de proveer a lo largo de la vida de las personas. O, mejor aún, oportunidades varias, que van desde la revisión de la red de establecimientos de Educación Superior existente, hasta la renovación e innovación de su oferta formativa, la armonización y comparabilidad en la designación de las titulaciones, la racionalización de los recursos y de su mejor aprove chamiento; e incluso, la posibilidad de situarse ante un nuevo paradig- 
ma en la formación y cualificación de la ciudadanía, y de lo que ambas deben representar para una mejor transición e inserción en la vida activa laboral, sobre todo de la población más joven.

Oportunidades también para la renovación del discurso educativo y de las prácticas en las que se proyecta, dentro y fuera de las instituciones escolares; en la educación formalizada por los contenidos curriculares y en la educación comunitaria, así como en la formación y especialización de quienes pretenden dedicarse profesionalmente a la educación, como maestros, profesores, educadores, pedagogos, psicopeda gogos, etc. Y, de un modo más o menos explícito, de los conocimientos y de las competencias que definen sus respectivos campos científico-disciplinares. Entre otros, y cada vez con mayor énfasis, todos aquellos que se identifican epistemológica, co n textual y experiencialmente con la Pedagogía Social y la Educación Social.

\section{La Reforma de Bolonia: un quehacer pedagógico y social para la construcción de la "nueva" Educación Superior en Europa}

Son abundantes los diagnósticos que coinciden en subrayar que la enseñanza superior está en crisis, que sus estudios carecen de suficiente calidad, o que sus contribuciones al desarrollo del conocimiento son limitadas y a menudo inconsistentes para responder a las demandas de la sociedad y del mercado laboral. De igual modo, son abundantes las pruebas que dan cuenta de su viabilidad a lo largo de los siglos y de su capacidad para transformarse y propiciar el bienestar de la sociedad, haciendo frente a problemas y desafíos de muy desigual alcance simbólico y material. Aunque siempre, en una dirección u otra, otorgándole a la palabra "reforma" un amplio recorrido semántico, en unas ocasiones profundamente refractario y conservador, en otras extremadamente alternativo y progresista. En general, y tras la excesiva utilización del término "reforma" para referirse a cualquier cambio acontecido en el interior de las universidades, dando pie a motivaciones y posicionamientos muy dispares.

Lo ilustró, como pocos, José Ortega y Gasset cuando, ante un público expectante, convocado por la Federación Escolar Universitaria Madrileña en el Paraninfo de la Universidad de Madrid, el 9 de octubre de 1930, al resumir su estado de ánimo ante las reformas académicas propuestas por aquel entonces para las Universidades españolas en una frase prodigiosa: "con mucho entusiasmo, pero sin mucha fe". Consciente de las críticas circunstancias que envo lvían la vida universitaria en los primeros años del siglo xx, muy lejos de lo que debían e incluso podían ser sus enseñanzas, no dudaba en reclamar temple y autenticidad para renovar los usos que las sustentaban; lo que a su juicio sólo podría llevarse a cabo acertando plenamente con su misión formativa, científica y cultural. Una misión con la que superar la falsedad y la desmoralización que inspiraba -según el crítico análisis del filósofo- todos los planes y estructuras de la universidad conocida, hasta el punto resultar ineludible vo l verla del revés, para reformarla radicalmente, partiendo de nuevos principios: "en vez de enseñar lo que, según un utópico deseo, debería ense ñarse, h ay que enseñar sólo lo que se puede enseñar; es decir, lo que se puede aprender..." (Ortega y Gasset, 1992, 43).

Para aquel Ortega, Europa y la Pedagogía constituían dos referentes clave para el cambio de rumbo que precisaban nuestras universidades. Y que hoy seguimos precisando, tal y como recordaba hace años Federico Mayor Zaragoza (1998, 309-310) en su condición de Director General de la UNES$\mathrm{CO}$, aludiendo a unos pocos conceptos, en 
los que se encierra -decía- una gran complejidad: la formación de ciudadanos conscientes, responsables y comprometidos; la capacitación de los profesionales que la sociedad necesita; el desarrollo de la investigación científica; la conservación y transmisión de la cultura, enriqueciéndola con el aporte creador de cada generación; la preservación de la memoria del pasado y la anticipación del futuro... erigida en "baluarte de los valores del espíritu y en gran animadora de un movimiento ético, que procure a la inteligencia el sentido de la solidaridad y el compromiso".

Europa, volvemos a Ortega (1992, 177), no sólo como un escenario consubstancial a la institución universitaria, con una trayectoria cuyos "altos y bajos, su humildad y su esplendor avanzaron paralelamente al entusiasmo que el europeo sintió por la inteligencia", sino también como un proyecto a construir con vocación de futuro, logrando que cada individuo o muchos individuos lleguen a sentirse partícipes de su "unidad vital". Cuando lo consiga -sentenciaría Ortega- la universidad volverá a ser lo que fue en su mejor momento: un principio promotor de la historia europea. Y, en su seno, como observa con mayor proximidad cronológica y social Edgar Morin (1998), un actor decisivo para el retorno a la problematización que inspiró la organización del pensamiento moderno y la misión social de la universidad, con importantes consecuencias existenciales, éticas y cívicas.

La Pedagogía era contemplada por Ortega y Gasset (1992: 49) como una cuestión capital al quehacer universitario, con principios y cometidos diferenciados de los que guían el cultivo de la cultura y de la ciencia; $y$, tal vez por ello, obligada a formular una advertencia principal: la actividad docente ha de partir no del saber ni del maestro sino del aprendiz, del estudiante. La universidad -afirma- "tiene que ser la proyección institucional del estudiante, cuyas dos dimensiones esen- ciales son: una, lo que él es: escasez de su facultad adquisitiva de saber; otra, lo que él necesita saber para vivir".

Como hemos expresado, transcurridas más de siete décadas, los anhelos orteguianos mantienen plena vigencia. $\mathrm{O}$ al menos eso parece, cuando Europa y la Pedagogía vuelven a ser los principales pretextos de las reformas que deberán afrontar nuestras Universidades, acomodando sus futuros desarrollos curriculares a la creación y mantenimiento de un Espacio Europeo de la Educación Superior cuyas principales coordenadas se inspiran en los acuerdos y declaraciones adoptados al más alto nivel gubernamental en La Sorbona (1998), Bolonia (1999), Praga (2001), Berlín (2003) o Bergen (2005), a la espera de la próxima reunión a celebrar en Londres en mayo de 2007. Un "espacio" al que se espera puedan vincular su labor docente, discente e investigadora cerca de 4.000 instituciones, con más de un millón de profesores y casi 15 millones de estudiantes, considerando el conjunto de países que integran actualmente la Unión Europea y los que están en proceso de adhesión. Todos ellos, de un modo u otro, expresando su firme voluntad de participar activamente en el logro de un propósito común: modernizar las universidades y sus enseñanzas como un componente indispensable para la consolidación y el enriquecimiento de la ciudadanía europea, afrontando los retos derivados de la sociedad del conocimiento, en un mundo abierto y en constante transformación.

La convergencia entre los sistemas nacionales de Educación Superior, procurando que como muy tarde en el año 2010 se alcance un desarrollo armonizado de las ofertas formativas de los distintos países que integran la Unión Europea, constituye el eje vertebrador de su previsible puesta en práctica, asumiendo -entre otras- la necesidad de emprender acciones conjuntas, destinadas a alcanzar seis objetivos principales: 
u Garantizar el acceso universal y continuo de las personas al aprendizaje, con el propósito de obtener y renovar las cualificaciones requeridas para participar de forma sostenida en la sociedad del conocimiento.

u Aumentar visiblemente la inversión en recursos humanos, para dar prioridad a los ciudadanos, a los que se considera el capital más importante de Europa.

u Desarrollar métodos innovadores y contextos eficaces de enseñanza y aprendizaje para alcanzar una formación continua a lo largo y ancho de la vida.

u Mejorar significativamente las formas en que se entienden y se valoran la participación en el aprendizaje y sus resultados, sobre todo en lo que atañe al aprendizaje no formal e informal.

u Lograr que todos los ciudadanos de Europa, a lo largo de toda su vida, puedan acceder fácilmente a una información y un asesoramiento de calidad acerca de las oportunidades del aprendizaje.

u Ofrecer oportunidades de aprendizaje permanente tan cerca de los interesados como sea posible, en sus propias comunidades y, cuando proceda, con el apoyo de las tecnologías de la comunicación.

Estos objetivos se proyectan en una serie de actuaciones prioritarias, con las que se pretende crear un sistema de grados académicos fácilmente comprensibles y comparables, fomentar la movilidad de los estudiantes, docentes e investigadores, garanti zar la calidad educativa y tener en cuenta la dimensión europea de la enseñanza superior. Y que se resumen en:

u Consolidar el sistema ECTS (European Credit Transfer System), como medio para reestructurar y desarrollar los currícula con el objetivode crear vías de aprendizaje flex ibles y centradas en el alumno. La implantación del sistema de créditos europeos agrupará toda la actividad académica de los alumnos, y se medirá en función del volumen de trabajo del estudiante (horas de clase, trabajos realizados dentro y fuera del aula, actividades académicas dirigidas, tiempo de estudio, etc.). Obliga no sólo a las Universidades y a los Centros de Enseñanza Superior a reconfigurar las materias que conforman el Plan de Estudios para estructurarse según esta nueva unidad de medida académica, sino también a replantear el papel y funciones docentes.

u Debatir y desarrollar definiciones comunes de marcos de titulaciones y de resultados del aprendizaje a nivel europeo, al mismo tiempo que se mantienen las ventajas de la diversidad y de la autonomía institucional en relación a cada currículum. La adaptación de las enseñanzas y títulos oficiales universitarios establece un sistema estructurado en dos títulos: un primer nivel de Grado, con objetivos formativos generales, de clara orientación profesional; y un segundo nivel de Post-grado (Master y Doctor), con un mayor grado de especialización. u Involucrar a académicos, profesores y estudiantes, organizaciones profesionales y empleadores en el nuevo diseño de los currícula para poder otorgar a los títulos de Grado y Master una entidad propia. El criterio de calidad y su acreditación deberán ser uno de los soportes fundamentales en la construcción del Espacio Europeo de la Educación Superior (EEES).

u Seguir definiendo y promocionando en el currículum las destrezas de capacidad de obtención de empleo en sentido amplio, y asegurarse de que los programas de primer ciclo ofrecen la opción de acceder al mundo laboral. El Suplemento Europeo al Título será, en este sentido, un documento anex o al título académico, en el que se reconocen los estudios realizados, las competencias profesionales, las capacidades y los conocimientos, formación complementaria, idiomas, etc. del alumno; su finalidad es fomentar el reconocimiento académico y profe- 
sional de las cualificaciones, favoreciendo así la movilidad no sólo en el ámbito universitario, sino también en el laboral.

Las transformaciones que se emprendan deberían configurar líneas básicas de actuación a observar en cada país y en el contexto europeo, de las que se espera tengan una importante repercusión en aspectos como: dar prioridad al aprendizaje, aprender a aprender, promover planes centrados en el alumno, contribuir a una formación integral, flexibilizar los aprendizajes, promover actividades diversas y complementarias, trabajar en equipo, etc.

Dejando al margen las discrepancias terminológicas que provocan algunas de las expresiones a las que se recurre, en la formulación de estos objetivos y actuaciones -en buena medida contenidos en el Memorandum sobre el Aprendizaje Permanente ela borado por la Comisión Europea y en el Marco de Acción aprobado por el Foro Mundial de la Educación de Dakar (2000)- se ponen de manifiesto las urgencias que motivan la búsqueda de nuevos horizontes para la formación de las personas y el desarrollo de las sociedades. Todo ello en un contexto socioeconómico, tecnológico y cultural que ha incrementando significativamente las necesidades y demandas de la población, subrayando -con palabras que hicieron suyas los participantes en la Conferencia Mundial sobre la Educación Superior en el siglo XXI (UNESCO, 1998)- que "los sistemas de educación superior deberían aumentar su capacidad para vivir en medio de la incertidumbre, para transformarse y provocar el cambio, para atender las necesidades sociales y fomentar la solidaridad y la igualdad; preservar y ejercer el rigor y la originalidad científicos con espíritu imparcial por ser un requisito previo decisivo para alcanzar y mantener un nivel indispensable de calidad; y colocar a los estudiantes en el primer plano de sus preocupaciones en la perspec- tiva de una educación a lo largo de toda la vida a fin de que se puedan integrar plenamente en la sociedad mundial del conocimiento del siglo que viene".

Un siglo en el que ya estamos y para el que, en menos de cinco años, las actuales titulaciones, y con ellas toda la estructura del sistema universitario español, serán revisadas en profundidad, con un alcance muy superior a cualquiera de las anteriores remodelaciones de los planes de estudio. Y no sólo porque el proceso afecte a más de cuarenta países, o porque el mapa de titulaciones (de grado y postgrado) se altere sustancialmente; sino también, y puede que de un modo decisivo, porque los cambios previstos están llamados a reconvertir la enseñanza en un proceso activo de aprendizaje, no limitado al aula, centrado en el trabajo individual y grupal de los estudiantes, a los que se convoca a participar en la discusión y construcción de su propio proyecto de formación. La organización de la enseñanza deberá centrarse, pues, en el alumno y en los objetivos de su formación, con un sentido diferencial y autónomo de lo que han de ser sus procesos de aprendizaje y la transferencia de las competencias que de ellos se deriven hacia la formación de ciudadanos comprometidos ética y moralmente con la sociedad actual y con la construcción de la ciudadanía europea (Martínez y Esteban, 2005).

Que sea así, al menos en las intenciones declaradas, refuerza el contenido pedagógico y social de lo que ha de ser la construcción del Espacio Europeo de la Educación Superior y de los principios en los que se sustenta, coincidentes con lo que tanto la Pedagogía Social (en su dimensión científica y normativa) como la Educación Social (en su praxis cotidiana y profesionalizadora) vienen reivindicando desde hace años: que los compromisos y responsabilidades educativas, allí donde se ejerzan (también en las universidades), deben posibilitar más y mejores logros en los derechos de la ciu- 
dadanía, conscientes de que es en ellos donde las personas y la sociedad en su conjunto se juegan su prioritaria razón de ser. Aunque también, donde la Pedagogía Social y la Educación Social -acaso comenzando una nueva etapa, como interpreta Jesús Vilar (2006) - afrontan con mayor trascendencia que nunca las pruebas con las que dar fe de sus respectivas identidades, es en los plano científico (como un saber que se crea), académico (como un saber que se transmite) y profesional (como un saber que se "aplica"). Tres perspectivas llamadas a integrarse, sin las que no es posible entender las relaciones y ubicaciones que se habiliten para la futura formación y profesionalización de los graduados en Pedagogía y Educación Social, en lo que tienen de común y en lo que se distinguen (Pérez Serrano, 2003; ANECA, 2005a).

\section{La Pedagogía Social y la Educación Social ante la convergencia europea: su estado de cuestión en el sistema universitario español}

En las tres últimas décadas, la paulatina incorporación a los Planes de Estudios de distintas universidades (Santiago de Compostela, UNED, Islas Baleares, Murcia, Autó noma de Barcelona, etc.) de especialidades o itinerarios formativos en los que se contemplaban contenidos con una clara proyección socio-educativa y pedagógica-social, condujeron a la progresiva y extensiva incorporación de la Diplomatura de "Educación Social" a nuestras Universidades, ya desde los primeros años noventa del pasado siglo, llegando a superar en número de centros y en alumnos matriculados a la titulación de Pedagogía.

No se trata, sin embargo, de una formación y profesión cuyos orígenes se remiten primordial y exclusivamente a las universidades, al menos en el caso español. Ya que, tal y como se expresa en el primer volumen del Libro Blanco del Título de Grado en Pedagogía y Educación Social, promovido por la ANECA (2005a), la titulación de Educación Social, aún siendo joven, tiene una larga trayectoria profesional, primero bajo la denominación de educadores especializados y, más tarde, como educadores sociales, a la que contribuyeron decisivamente -entre otras iniciativas- la creación, en 1972, de la primera Asociación de Educación Especializada en Barcelona y la celebración de un primer congreso en 1987.

Con todo, no se puede obviar que la definitiva expansión e institucionalización de la Educación Social (y, en relación a ella, de la Pedagogía Social) se produce a raíz de las propuestas emanadas de los Informes Técnicos del Grupo 15, relativos a las directrices generales propias de los Títulos de "Diplomado en Educación Social" y "Licenciado en Educación Social”, elevados al Pleno del Consejo de Universidades a finales de los años ochenta, y con los que se abrió un proceso de debate, tras el que se determinaría la creación de las enseñanzas conducentes a los títulos oficiales de "Diplomado en Educación Social” (Real Decreto 1420/1991, de 30 de agosto; BOE del 10 de octubre de 1991) y de "Licenciado en Pedagogía" (Real Decreto 915/1992, de 17 de julio de 1992; BOE de 27 de agosto de 1992).

En la propuesta del Grupo 15, en noviembre de 1987, se justificaba la decisión de crear el título de Diplomado en Educación Social en los siguientes términos: "necesidades sociales de diverso tipo, el que la educación y la formación actúen a través de nuevos canales y medios, las necesidades de la educación relacionadas con el mundo del ocio, así como el reconocimiento de que la formación es una necesidad durante toda la vida activa de los sujetos, e incluso fuera de ella, han planteado la necesidad de considerar el ámbito de la educación no formal y fuera de las instituciones escolares como un campo en el que existe una demanda 
social de personal específicamente formado para ellas que en la actualidad no atiende la oferta actual de títulos universitarios".

Las enseñanzas de este primer ciclo universitario de Educación Social, a juicio de la Comisión, deberían estructurarse sobre tres pilares básicos: la educación de adultos, entendida como actividad más amplia que la alfabetización y la recuperación hasta convertirse en educación permanente; la formación laboral, ya que dadas las características del mundo cambiante del trabajo y la renovación acelerada de las técnicas de producción, se ha desarrollado todo un ámbito de necesidades de formación relacionadas con el empleo; y la educación de sectores marginales de la sociedad, que requiere adecuar tratamientos educativos específicos en orden a la recuperación de los individuos afectados y a la prevención de la misma marginación. Consecuentemente, las enseñanzas del Diploma estarían "orientadas a la formación de un educador que actúa en ambientes no escolares, en atención preventiva y de recuperación con jóvenes marginados, así como en la acción socio-educativa en ambientes naturales, inserción de jóvenes en la vida adulta, etc.".

Para una duración estimada de las enseñanzas de 3 años, con una carga lectiva comprendida entre un mínimo de 180 créditos y un máximo de 270, se proponen como materias troncales: Antropología Social, Educación Permanente, Historia Social de la Educación, Métodos de Investigación Socioeducativos, Practicum, Programas de Animación Sociocultural, Psicología Evolutiva, Psicología Social y de las Organizaciones, Psicología infantil y juvenil, Sociología y Teoría de la Educación.

Partiendo de esta propuesta y de las revisiones (contextuales y hasta legales) que de ella han ido haciendo las universidades españolas, los Planes de Estudio de la Diplomatura en Educación se desarrollan curricularmente en 36 instituciones universitarias (curso académico 2003-04), con una gran heterogeneidad en cuanto a su contenidos y perfiles formativos, tal y como se refleja en el Libro Blanco de la Titulación, elaborado por la Red de Educación y editado por la ANECA (2005a). Esto se debe, en gran medida a la elevada presencia de créditos destinados a materias obligatorias de Universidad y a las materias optativas ofertadas por cada centro, valorándose como un elemento positivo de este hecho la "amplia diversidad de formación que encontramos entre las propuestas de cada Universidad"; mientras que, como aspecto negativo, se apunta que "esta misma diversidad tanto en contenidos como en asignación de créditos ha entorpecido seriamente las posibilidades de convalidación de planes de estudio entre las Universidades españolas". No obstante, sólo cuatro de ellas ofertaban itinerarios especializados: el CSEU la Salle, adscrito a la Universidad Autónoma de Madrid; la Universidad de Lleida; la Universidad Pablo de Olavide de Sevilla; y la Universidad de Santiago de Compostela. Ya entonces, tanto la Escuela Universitaria Pere Tarrés (en la Universidad Ramón Llull) y la Universidad Pablo de Olavide, ofrecían la doble titulación en Educación Social y Trabajo Social.

En todo caso, se concluye que la demanda de sus estudios supera en gran medida la oferta que realizan las instituciones universitarias, siendo "una titulación que goza de gran aceptación, que refleja perfectamente un nuevo yacimiento de empleo, expresión clara de las necesidades específicas de la sociedad actual". A lo que se añade que "la mayoría de estas universidades ofertan a sus alumnos el acceso a un segundo ciclo en el mismo centro universitario", en una clara alusión a las titulaciones de Pedagogía y Psicopedagogía. De este modo, por vez primera, los cambios cuantitativos y normativo s que afectan a estas tres titulaciones acabaron proyectándose en transformaciones cualitativas de indudable trascendencia cientí- 
fica, profesional y social para las Ciencias de la Educación en general y, para la Pedagogía en particular, tanto en el plano docente como en el investigador, no sólo en relación al inmediato pasado, sino también de lo que han de ser sus desarrollos futuros en el marco de la Convergencia Europea de la Educación Superior.

En las universidades, especialmente en las Facultades de Ciencias de la Educación, los cambios que se producen generan nuevos intereses y expectativas en relación con el desarrollo de los estudios pedagógicos en España, cuando menos en dos perspectivas: de un lado, la que coincide en valorar su importancia para una renovada y más sugerente formación "profesional" de los educadores y de los pedagogos; de otro, la que acepta el desafío que supone comprometer la docencia universitaria con un esfuerzo específico de adaptación de las enseñanzas pedagógicas a las nuevas exigencias formativas y profesionales de la educación (Muñoz Sedano, 1994), hasta el momento limitadas casi en exclusiva a la formación de maestros, pedagogos y psicopedagogos que desempeñan su labor en el subsistema escolar.

$\mathrm{Al}$ respecto, no debe olvidarse que en las universidades ya se habían anticipado algunas de las preocupaciones esenciales acerca de lo que debería y podría hacer esta formación en la medida en que fuese contemplada y desarrollada en la reforma de las titulaciones y planes de estudios, ya desde los últimos años ochenta del pasado siglo. De ello quedó constancia, entre otros acontecimientos, en la celebración de las IV Jornadas Nacionales de Pedagogía Social monográficamente dedicadas a la "formación y profesionalización en Educación Social” (Santiago de Compostela, septiembre de 1987); y en las Jornadas sobre la Formación de Educadores y Agentes Socioculturales (Barcelona, abril de 1988), en las que se aprobó un extenso e interesante "documento final", en cuyo texto se recogían las "propuestas alternativas, observacionesy sugerencias formuladas al Informe Técnico durante el período de información y debate públicos" al Título de Diplomado en Educación Social; o el Congreso sobre la Educación Social en España, convocado por la Universidad de Comillas y la Fundación Santamaría (Madrid, septiembre de 1989).

Tampoco puede pasarse por alto que, una vez implantadas las enseñanzas conducentes a los títulos de Educación Social y de Pedagogía, siguieron activándose importantes foros de debate (Congresos, Jornadas, Simposios, Seminarios, etc.) en toda la geografía española, ibérica e iberoamericana; en muchos de ellos aunando la iniciativa institucional (esencialmente de orden científico y académico) de las universidades con la estimable e incesante capacidad de convocatoria de la que se dotan las Asociaciones y los Colegios Profesionales de los Educadores y Educadoras Sociales

También en clave académica e investigadora, serán especialmente significativos los Seminarios Interuniversitarios de Pedagogía Social, que se celebran ininterrumpidamente cada año desde 1985 -después de las I y II Jornadas, en Sevilla y Santiago de Compostela respectivamente, en las que la convocatoria atendía a la denominación conjunta de "Pedagogía Social y Sociología de la Educación"-, cuyas ediciones de 1985 (Murcia), 1987 (Santiago de Compostela), 1991 (Sevilla), 1994 (Valencia), 1995 (Madrid), 1999 (Burgos) y 2002 (Salamanca) tendrían como tema monográfico cuestiones relacionadas con la formación y la profesionalización en Pedagogía-Educación Social. La celebración en Santiago de Chile, mediante convocatoria conjunta por parte de la Unive rsidad Mayor y la Sociedad Iberoamericana de Pedagogía Social (en la que se integró y renovó la extinta Sociedad Ibérica de Pedagogía Social), en noviembre de 2004, del I Congreso Iberoamericano de Pedagogía Social, alentó nuevas perspectivas para el futuro desarrollo académico y profesional 
de la Educación Social en España, Portugal y América Latina, ante lo que ha de ser -en los próximos años- la construcción de la convergencia en la Educación Superior y la Investigación en el espacio iberoamericano.

Aunque, en muchos casos, son acontecimientos que traspasan las fronteras de lo académico-curricular, todos ellos han incidido decisivamente en la institucionalización de la Pedagogía Social y de la Educación Social en nuestro país, en Europa y América Latina, sin parangón con cualquier tiempo histórico precedente. Al menos en dos líneas de actuación claramente definidas: de un lado, la que permite constatar la creciente pluralidad epistemológica, metodológica y práxica del quehacer pedagógicosocial y de su proyección en la sociedad; de otro, la que pone de relieve la continua renovación e innovación de las comunidades científicas, académicas y profesionales que trabajan en este campo, sea cual sea el ámbito de la teoría y/o de la práctica socioeducativa en la que nos situemos.

Nos quedamos, en este sentido, con los juicios emitidos por el profesor José Ortega (1999: 38), cuando recuerdan cómo con “la creación de la titulación universitaria de primer ciclo de Diplomado en Educación Social se establecen las condiciones que posibilitan en principio una formación más sistemática, reglada y rigurosa del educador social. Se salía al paso de una cierta confusión, se introducía una mayor clarificación conceptual y científica, se abrían caminos a la investigación, a la reglamentación y dignificación de la profesión. En cualquier caso, tanto profesionales de la educación en la práctica como estudiosos de los diferentes aspectos de la educación social, creían, y entiendo que siguen creyendo, que era y es un paso necesario y conveniente esa creación de la Diplomatura en Educación Social en el marco de la Universidad".

También el emitido por el profesor Martí March (1998: 31), cuando señala que "la cre- ación de la diplomatura, a pesar de sus insuficiencias y deficiencias, no sólo supone el reconocimiento de una historia, de una realidad y de una profesión, sino también la posibilidad de construir esta profesión de una forma más viva, dinámica y permanente". A lo que añade: "si hasta la aprobación de la Diplomatura en Educación Social, este profesional ha tenido una formación plural, contradictoria y ajena a la universidad, resulta evidente que a partir de la misma aprobación dicha formación se plantea desde un enfoque homogéneo a base de criterios de contenido, de espacios de formación, de articulación interna del mismo, de ámbitos de prácticas, de reconocimiento social y profesional con la posibilidad de la existencia de un Colegio Profesional, etc.".

O, más recientemente, por los profesores Pla nelles y Vilar (2006: 13), cuando expresan que "la pedagogía social ha desempeñado un importante papel en la reconceptualización de las prácticas socioeducativas de finales del siglo XX y principios del siglo XXI. A lo largo de una trayectoria (desigual pero, al fin y al cabo, constituida y configurada con buenos cimientos teóricos), ha navegado por diferentes océanos temáticos y ha anclado en distintos puertos que le han permitido construirse como saber y como disciplina. Prueba de este itinerario -de construcción como saber y como disciplina- son algunos de los trabajos monográficos $\mathrm{d}$ e dicados a investigar con la finalidad de situar académicamente la pedagogía social". De aquí parten la Pedagogía Social y la Educación Social en las universidades españolas hacia la búsqueda de nuevas convergencias académicas, disciplinares y profesionales con las Universidades europeas. Para un trayecto abierto a múltiples contingencias, y en el que, junto a otros significados de naturaleza contextual y prospectiva, también comporta un cierto retorno a sus orígenes intelectuales y escolásticos, al menos en la tradición centroeuropea y mediterránea, y de forma particular en Alemania e Italia. 


\section{Realidades y desafíos de la Pedagogía Social en la Europa del siglo xxı: por una sociedad educadora, a favor de la condición ciudadana}

La expansión e institucionalización académica de la Pedagogía Social en un mundo cada vez más globalizado, en el que como ha expresado Castells (1997, I: 27) estamos abocados a una ostensible transformación del "paisaje social de la vida humana", del que participan un complejo e imprevisible mosaico de escenarios, no pueden entenderse al margen de las múltiples realidades, problemáticas y desafíos de nuestro tiempo. Y de un modo especialmente relevante de aquellos que conciernen, por defecto o por exceso, a las derechos humanos, a la condición ciudadana, a los valores democráticos, a las convicciones morales y culturales y, en general, a los procesos de desarrollo social, a la dignidad de las personas y de los pueblos y a su legítima aspiración a una mejor calidad de vida, con criterios de justicia y equidad.

En verdad, nada de lo que señalamos queda fuera de las preocupaciones e intenciones a las que se remite la educación, entendida en su perspectiva más integral y permanente, en los últimos años, al hacer explícito su deseo de contribuir al bienestar individual y colectivo, a mejorar la calidad de vida de los ciudadanos y a fomentar su igualdad de oportunidades. En cualquier caso, haciendo del desarrollo sostenible un asunto de todos, proclamando que el cambio hacia una mejor calidad de vida empieza por la educación, y que -tal y como se refrendó, en diciembre de 2002 por la Asamblea General de las Naciones Unidas en su resolución 57/254, relativa al Decenio de las Naciones Unidas de la Educación para el Desarrollo Sostenible (2005-2014)- trabajar a favor de esta educación no es una opción, sino una prioridad. Dentro y fuera del sistema educativo, en los curricula escolares y en todo tipo de formación, en los programas individualizados y en la acción comunitaria, en las políticas públicas y en la iniciativa cívica, con la intensa carga explicativa y comprehensiva que fundamenta los cuatro pilares básicos de una educación para todos y con todos: aprender a conocer, aprender a hacer, aprender a convivir y aprender a ser (Delors, 1996). Y ahí han de estar, con sus protagonismos diferenciados, si bien con propósitos confluyentes, la Pedagogía Social y la Educación Social.

También lo han de estar en la construcción europea y en los avatares que envuelven su complicada"constitucionalización", en un proceso de idas y vueltas, ahora mismo estancado, al que sin embargo no puede negársele sus dosis de realidad (Del Río, 2004): por su naturaleza y significados, por la forma de abordar la tarea, por la voluntad y por su visibilidad. Y también por el objeto: una Constitución; y por el sujeto: una Convención democrática y representativa, una sociedad llamada -aunque tan sólo sea formalmente- a participar. Algo que sólo podrá hacerse mediante la apropiación y el pleno ejercicio de los derechos que tiene reconocidos y legalmente habilitados sus ciudadanos. Porque, como subraya Aldecoa (2004: 20), "con la Constitución el ciudadano europeo encuentra su lugar en la Unión. Primero como legitimador de la misma. Segundo, encontrando un límite al poder público europeo derivado del respeto a sus derechos. Tercero, encontrando un vínculo político directo con la Unión como ciudadano de la misma. Y cuarto, teniendo en cuenta la opinión de los ciudadanos asociados para mejor definir y aplicar las políticas que les van dirigidas e, incluso, el derecho de los ciudadanos de pedir leyes concretas de la Unión". En las derivaciones políticas, estratégicas y "prácticas" de estos logros, la Pedagogía Social y la Educación Social también deben ocupar un lugar relevante y apreciable. 
Y deberán hacerlo en muchos frentes, aunque poniendo sobre todo el acento en la promoción y el desarrollo de los nuevos modelos educativos y sociales que se precisan para una construcción europea de amplias miras, tanto en sus perfiles pedagógicos (aspirando decididamente al logro de una auténtica sociedad del conocimiento y del aprendizaje, educante y educadora por sus métodos y finalidades) como en sus connotaciones cívico-sociales, definitorias de la condición ciudadana; entendida ésta como el mayor logro de toda politeyademocrática avanzada y de cualquier sociedad civil republicana habitada por gentes libres (Giner, 2000 y 2005).

En la vertiente pedagógica, los retos que la Pedagogía-Educación Social tendrá que hacer suyos en el plano científico, académico y profesional -es decir, allí donde sus líneas de investigación, los planes de estudio y la formación de sus titulados han de situar sus respectivas prioridades en el proceso de la Convergencia Europea de la Educación Superior-, pasan por reforzar su contribución al aprendizaje permanente, en cualquier tiempo y lugar, adscribiendo sus actuaciones a los objetivos e iniciativas que se contemplan en el "Programa Integrado de Acción en el ámbito del Aprendizaje Permanente"; una Propuesta de Decisión del Parlamento Europeo y del Consejo con fech a del 14 de julio de 2004. Si bien, haciendo notar que tales actuaciones no tienen porqué identificar a las personas como "alumnos" o "estudiantes" que esperan obtener un diploma, sino como sujetos (niños, jóvenes, adultos, mayores) que participan de un quehacer educativo mediante el que se procura acrecentar su realización personal, la cohesión social, la ciudadanía activa, la igualdad entre hombres y mujeres, la participación democrática, la animación cultural, el desarrollo comunitario, los valores ecológicos o su cualificación laboral. Tareas, todas ellas, que complementan y diversifican las res- ponsabilidades formativas de los sistemas educativos nacionales (desde la Educación Infantil hasta las universidades) en una construcción más plena de la biografía de cada persona y de las relaciones que hacen posible su convivencia con las demás.

En las coordenadas cívicas y sociales, el protagonismo lo detentan las políticas sociales y lo que ha dado en llamarse el "modelo social europeo". En los propios documentos de la Comisión Europea (2000), hace años que se admite que el objetivo de aquella reside en promover una calidad y un nivel de vida apropiados para todos en una sociedad activa, diversa y sana, en la que las mejoras de la productividad y del crecimiento económico deben revertir en una firme solidaridad entre los ciudadanos, como soporte indispensable para crear una sociedad estable y con unos mínimos niveles de bienestar. Recuerda Susana del Río (2004: 134) que ésta no ha sido ni está siendo una tarea fácil, ya que no basta con que en la configuración del modelo social europeo se reconozca que las personas y la sociedad civil son los actores indiscutibles, "tanto desde la dimensión impulsora en el proceso, como desde su significado intrínseco". Además, y como no podrá ser de otro modo, se precisa de un alto grado de concienciación e implicación cívica, que sólo puede garantizar una educación a la que se dote de una clara vocación política y social. Porque tal y como ha explica do Pascal Fontaine (2003: 43), profesor del Instituto de Estudios Políticos de París y antiguo colaborador de Jean Monnet, "el sentimiento de pertenecer a una misma colectividad, de compartir el mismo destino, no puede crearse artificialmente. De ahora en adelante, la Europa cultural debe tomar el relevo de la Europa económica y contribuir a la formación de una conciencia común".

El papel estratégico que, en el mejor de los sentidos, le corresponde desempeñar a la educación y a la formación en la construcción del Espacio Social Europeo, puede 
y debe tener en la Pedagogía Social -un campo de conocimiento al que se le demanda ocuparse de una profesión, la Educación Social, cada vez más visible en nuestra sociedad (Sáez y García Molina, 2006)- uno de sus principales baluartes académicos y disciplinares, en clave científica e histórica (Caride, 2005). Y, consecuentemente, un soporte epistemológico, conceptual, teórico y metodológico en el que sustentar el desempeño profesional de los pedagogos y de los educadores sociales. E, incluso, si las enseñanzas alcanzasen el nivel de transversali dad e interdisciplinariedad que se reclama para mejorar la Educación Superior a lo largo del siglo XXI, el de otros profesionales sociales y de la educación.

\section{Las lecciones aprendidas: apuntes para una visión prospectiva de la Educación Social y de su Pedagogía en el Espacio Europeo de la Educación Superior}

En España, la experiencia formativa acumulada en la Diplomatura en Educación Social, a tenor de los informes evaluativos -internos y externos- que se han ido realizando en los últimos años en muchos de los Centros y Universidades que tienen implantada la titulación, pone de relieve algunas de las debilidades y fortalezas derivadas de su incorporación a las enseñanzas universitarias; y que, a modo de lecciones "aprendidas" o, al menos "escritas", aluden a cuestiones clave de su pasado y, en buena medida, de lo que deberá ser su futuro, como son: el diseño de sus planes de estudios, la organización y coordinación de la oferta formativa, el perfil teórico y práctico de las enseñanzas que imparten, la transición e inserción de los titulados en el mercado laboral, etc. Entre las debilidades detectadas, cabe destacar las siguientes:

u El todavía insuficiente conocimiento de la titulación por parte de sus potenciales estu- diantes, desvelándose la poca o muy escasa información que sobre ella poseen los alumnos que cursan la Enseñanza Secundaria (Bachillerato).

u La equívoca e imprecisa caracterización que de la Educación Social y de la formación del educador social se hacía en el Real Decreto $1420 / 1991$, mediante el que se creaba la titulación y se regulaban las enseñanzas conducentes a la obtención del título oficial de Diplomado en Educación Social, en ocasiones haciendo uso de expresiones que contravienen el amplio recorrido contextual, teórico-conceptual, epistemológico, pedagó gico metodológico y profesional de la Educación Social.

u La indefinición, y en algunos casos el solapamiento o la tergiversación, que se constata en algunos Centros y Planes de Estudios de lo que debería ser la filosofía del Título y el desarrollo de teórico-práctico de sus enseñanzas y aprendizajes, al estar excesivamente marcados por cuestiones como: la tradición pedagógica-docente-escolar (o, si se prefiere, por una lectura "escolar" de la educación); los desajustes que se producen entre los contenidos teóricos y las prácticas; la falta de correspondencia entre los conocimientos fundamentantes y los que tienen una orientación más especializada; la baja articulación interdisciplinar entre algunas materias y las áreas de conocimiento responsabilizadas de su impartición; el desajuste que existe entre la troncalidad -e incluso entre algunas materias optativas-y los ámbitos profesionales de la Educación Social; las limitaciones que todavía presenta la realización del practicum en aspectos clave como son la regulación plena de todas las condiciones que lo afectan en los convenios que se suscriben con otras instituciones, el reconocimiento de la labor tutorial-docente de los profesionales que en ellas trabajan, etc.

u La incongruencia observada entre los procesos y metodologías de enseñanza-aprendizaje que se requieren para el desarrollo de

[ 24 ] SIPS - PEDAGOGÍA SOCIAL (ISSN-1139-1723) № 14 TERCERA ÉPOCA 
la actividad docente-discente (innovadora y necesariamente interactiva en muchas de sus propuestas y prácticas, en los términos en que parece exigido por una titulación de perfil educativo-social) y las realidades que concretan tal actividad en el aula, manteniéndose planteamientos muy convencionales tanto en los métodos didácticos como en los sistemas de evaluación (dictado de apuntes, explicación centrada en el profesor, exámenes tradicionales, etc.). Y que, en general, no sólo limitan la participación del alumnado y su motivación hacia la carrera, sino que también, con visión de futuro, alientan actitudes contradictorias respecto de lo que deberá ser su plena inserción e implicación en las prácticas profesionales que desempeñen.

u El insuficiente reconocimiento y valora ción de la titulación en las realidades circundantes, internas y externas, a las propias Universidades; y, muy especialmente, en aquellos escenarios laborales llamados a soportar buen parte del ejercicio profesional de los educadores sociales, mu chos de ellos dependientes de la las Administraciones Públicas Locales o Autonómicas. Una cuestión que no cabe atribuir en exclusivaa la emergente profesionalización e inserción laboral de los titulados en Educación Social, de sus funciones y competencias, sino al desinterés de las propias instituciones, o a prácticas que perpetúan la falta de políticas educativas y sociales consistentes, el corporativismo o la contratación precaria.

Entre sus puntos fuertes, otras veces llamadas fortalezas o potencialidades, cabe destacar los siguientes:

u Se trata de una Titulación que a pesar de su reciente creación e implantación goza de un notable reconocimiento académico y profesional, que no ha dejado de acrecentarse en los últimos años, ampliando sus ofertas formativas (públicas y privadas) en las Uni- versidades; introduciendo cambios importantes en la organización de sus enseñanzas, aprovechando las reformas parciales de sus Planes de Estudios; en la diversificación y mejora del practicum, en la difusión y publicidad de sus estudios; en la cooperación entre las universidades y las Asociaciones o Colegios Profesionales, etc.

Tal y como se subraya en la mayoría de las aportaciones que realizan los ponentes que participaron en las Jornadas de Estudio y debate sobre el Grado en Educación Social - celebradas en Palma de Mallorca en junio de 2006, por iniciativa de la Conferencia de Decanos y Directores de Centros con títulos de Maestro y de Educación, con la colaboración de la Sociedad Iberoamericana de Pedagogía Social (SIPS)-, estamos ante una titulación que ha ido acumulado experiencia, con un bagaje significativo de realizaciones, en general positivo y del que no puede prescindirse de cara a lo que podrá y deberá ser su optimización en el Espacio Europeo de la Educación Superior.

u La capacidad de respuesta que ofrece la Titulación, por su polivalencia y fácil acomodación a diferentes campos de especialización, a necesidades sociales, culturales, profesionales, laborales, etc. emergentes en la sociedad, en confluencia con lo que acontece en otros países y con las expectativas de los estudiantes que la cursan; muchos de los cuales optan por cursarla con una decidida vocación profesional, con una demanda que supera en gran medida la oferta de las instituciones universitarias, no siendo significativa la diferencia entre la primera y la segunda opción en la matrícula, tal y como se refleja en el Libro Blanco editado por la ANECA (2005a). En definitiva, porque se trata de unos estudios que han logrado articular positivamente buena parte de sus cimientos teoréticos con su inequívoco perfil profesionalizador; por lo demás, bien identificado.

u La creciente formación y, en algunos casos, especialización docente e investigadora que 
se está produciendo en el campo, ya sea a través de las fuentes de conocimiento más específicas que procura la Pedagogía Social, ya sea a través de otros ámbitos y saberes de la Educación y de la Acción Social en general. Se trata, no se puede obviar, de una titulación que promueve una formación universitaria polivalente en el ámbito de las Ciencias de la Educación, con una configuración académica a la que se vinculan diversos Departamentos y Áreas de Conocimiento, tanto en las materias troncales y obligatorias como en las optativas, lo que ayuda a percibir sus estudios como un espacio para el saber compartido y el quehacer común.

Debe significarse que a esta dinámica están respondiendo también muchas de las actuaciones que se promueven en el mundo editorial (con colecciones o secciones bibliográficas que aluden expresamente a la Peda gogía Social o a la Educación Social); en las publicaciones periódicas (edición de nuevas revistas especializadas o de monográficos en las revistas que gozan de cierta tradición e impacto científico); en la convocatoria de Jornadas, Congresos, Simposios, Seminarios, Reuniones, etc. que con carácter internacional, nacional, autonómico y local, se vienen celebrando en las dos últimas décadas, mostrando el potencial científico y disciplinar de la Educación Social en distintos contextos.

u La creación y puesta en funcionamiento de distintas iniciativas orientadas a una mejor y mayor transición desde la formación al desempeño profesional. De un lado, son actuaciones que suelen repercutir en la capacidad de iniciativa e innovación puesta de manifiesto por las universidades y los centros de formación; pero, de otro, y sobre todo, revelan la alta capacidad de autoorganización que poseen los colectivos profesionales de educadores y educadoras sociales, a través de sus Asociaciones y Colegios Profesionales, a menudo mediante acciones concertadas con las Universidades, para estrechar las vías de colaboración existentes con las Administra ciones Públicas (sobre todo autonómicas y locales) y los agentes sociales (organizaciones, fundaciones, asociaciones, et c.) potencialmente generadoras de empleabilidad. O, en su defecto, de iniciativas formativas (cursos de especialización, masters, etc.) que contribuyan a una mayor adecuación de la formación recibida a los puestos de trabajo ofertados.

Sin que se agoten en esta doble lectura ("debilidades" versus "fortalezas"), las opciones de análisis, todo indica que es preciso tener en cuenta distintas recomendaciones de carácter prospectivo, especialmente si se tiene en cuenta que muchas de ellas son propuestas de mejora emanadas de los Comités de Evaluación -internos y externos- que ejercieron su labor en un buen número de Universidades españolas desde mediados de los años n oventa hasta la actualidad. De un modo u otro, son referencias "prácticas" y "estratégi cas" para la incorporación del Grado de Educación Social al Espacio Europeo de la Educación Superior, y en su interior de los contenidos científicos y disciplinares que podrá aportar a su desarrollo la Pedagogía Social.

Cuando todavía esperamos la redacción de las directrices para la elaboración de Títu los de Grado por Ramas de Conocimiento (Artes y Humanidades, Ciencias, Ciencias de la Salud, Ciencias Sociales y Jurídicas e Ingeniería y Arquitectura), vinculadas a grandes familias disciplinares, todo indica que el futuro Grado en Educación Social debe tener muy en cuenta las contribuciones realizadas por distintos colectivos e informes; entre otros, el Libro Blanco editado por la ANECA (2005a) y los documentos redactados para las Jorna das de Estudio y Debate sobre el Título de Grado en Educación Social (Palma de Mallorca, junio de 2006), a las que ya hemos aludido, a partir de cuya celebración se confeccionó una "Ficha Técnica de propuesta de título universitario de Grado en Educación Social”, 
atendiendo a las consideraciones que sobre su elaboración figuraban en el Real Decreto 55/2005, de 21 de enero, que en aquel entonces regulaba los estudios universitarios oficiales de Grado, al tiempo que establecía la estructura de las enseñanzas universitarias; ahora sometidas a revisión.

Ante un futuro incierto, al que se irán prendiendo nuevas disposiciones normativas y acuerdos en la política universitaria y en el seno de las universidades, mantene mos el interés que ciertas propuestas tienen para tomar decisiones que favorezcan el desarrollo académico y profesional de la Educación Social en el Espacio Europeo de la Educación Superior, y también de la Peda gogía Social como su principal y más sustantivo referente científico. Y que podemos concretar en:

u Mejorar la correspondencia existente entre la denominación de la Titulación, los objetivos que se pretenden y los contenidos que se imparten, tanto desde una perspectiva académica como profesional.

u Reforzar la articulación y coordinación (vertical-horizontal) de las materias que integran el curriculum, tanto desde su organización docente (en las Áreas y Departamentos que corresponda) como discente (en la articulación de los saberes teóricos y prácticos con los que trabajen los estudiantes). u Promover, a través de distintas vías, acti vidades de innovación pedagógica que impliquen la revisión de las metodologías de trabajo en el aula, la introducción de nuevas tecnologías en los procesos de enseñanza-aprendizaje, la interacción de la forma ción teórica y práctica, etc.

u Crear o activar redes de cooperación institucional que posibiliten una mejor transición desde la formación inicial (Grado) a la formación continua (postgrado), de la formación polivalente a la formación especializada, de la formación teórica a la formación práctica (en el propio centro formativo y en otros contextos, fundamentalmente mediante el practicum o los programas de movilidad), de las realidades próximas (municipales y autonómicas) a las nacionales e internacionales, especialmente en los contextos europeo y latino-americano.

u Participar activamente, desde la formación y/o desde el quehacer social (ante las Administraciones Públicas, las entidades empleadoras, etc.), en la definición del perfil profesional de los titulados, de su identidad y estatuto, como elementos imprescindibles en la mejora de la imagen social y de la proyección socio-profesional de estos estudios y de quienes se capacitan en ellos. En este sentido será importante mejorar, hasta donde sea posible, la definición de sus competencias y de las funciones que les corresponde desempeñar respecto de otros profesionales sociales y de la educación, evitando solapamientos o duplicidades entre ellos.

En relación a este punto, es importante subrayar la necesidad, por coherencia social, insti tucional, académica y profesional, de que los Grados de Pedagogía y de Educación Social tengan perfiles claramente diferenciados. De hecho, así cabe deducirlo de los objetivos que se formulan para uno y otro título, de buena parte de sus respectivos bloques de contenido y de una parte considerable de los ámbitos y perfiles profesionales que se han diseñado para cada titulación. Con todo, es aquí donde se precisa un mayor esfuerzo de clarificación, reduciendo o eliminando las convergencias que se establecen entre ambos Grados en perfiles como:

u Formador de personas adultas y mayores, que seguimos estimando que se trata de un perfil en el que tradicionalmente, y con la denominación de "educador de adultos", se ha venido trabajando en la Educación Social. u Educador Ambiental, cuya denominación y también por tradición del campo se sitúa claramente en los ámbitos de la Educación Social. Que esto sea así no significa que desde el que- 
hacer científico, disciplinar e incluso profesional de la Pedagogía no se participe -con criterio de interdisciplinariedad y multiprofesionalidad- en programas y acciones educativas que tengan como soporte la formación y educación de personas adultas (entre ellas, los mayores) o la Educación Ambiental; o que, como será deseable, en los Postgrados (Master y Doctorado) que se creen, no se prevean confluencias entre ambas titulaciones.

Por lo que se refiere a la propuesta del Título de Grado en Trabajo Social, el Libro Blanco editado por la Agencia Nacional de Evaluación de la Calidad y AcreditaciónANECA (2005b: 106) no obvia que una de las áreas profesionales de tal titulación, en el marco de la "intervención indirecta", reside en la labor "promocional-educativa", seña lando que "no se puede comprender la función de prevención sin una labor educacional que ayude a las personas y los grupos sociales a hacer uso de las oportunidades que existen a su disposición. Capacitarles para la toma de decisiones y asunción de responsabilidades (se considera una condición transversal)".

Tampoco puede obviarse que al ser definido el/la trabajador/a social como "un/a profesional de la acción social que tiene una comprensión amplia de las estructuras y procesos sociales, el cambio social y del comportamiento humano" que le capacita para la "integración social de personas, familias, grupos, organizaciones y comunidades, (...) la constitución de una sociedad cohesionada" y "el desarrollo de la calidad de vida y del bienestar social"... existan notables puntos de convergencia académica, disciplinar y profesional con la Educación Social y con los/las educadores/as sociales. Lo que, por el bien de la ciudadanía y de un mayor y mejor desarrollo de las políticas sociales, estima mos como positivo... máxime cuando en el propio documento (ANECA, 2005b: 111) se considera que tales fines han de ser logrados mediante la contribución con "otros pro- fesionales de la acción social".

Al respecto, no hay mucho que objetar a que cuando se delimitan los ámbitos de intervención de los trabajadores sociales, en el apartado referido a "servicios sociales especializados (atención por colectivos)", se mencione la educación, en dos niveles (ANECA, 2005b: 142):

u Reglada: servicios de asesoramiento psicopedagógico y social a los equipos de los centros escolares e institutos de enseñanza secundaria; mediación escolar y familiar; centros de educación especial para alumnos con necesidades especiales; asociacionismo en el ámbito escolar; detección y tratamiento del absentismo escolar.

u No reglada: escuelas-taller y otras iniciativas orientadas a compensar déficits socioeducativos; servicios de atención a primera infancia, especialmente con familias que requieran soporte psico-social.

Con relación a esta propuesta, nos detenemos en consideraciones principales: la primera, establece una profunda discrepancia con la denominación de tales ámbitos, máxime cuando se podría haber optado por una diferenciación más acorde con la terminología al uso, por ejemplo: educación escolar y educación social. La segunda, un cuestionamiento radical de la propuesta si en el marco de sus contenidos (comunes obligatorios y optativos o de universidad) no se incorpora el ámbito disciplinar de la Peda gogía. De momento, en los primeros (contenidos comunes obligatorios), en el Libro Blanco del Grado sólo se contemplan:

u De un lado, el Trabajo Social (teoría y práctica), aludiendo expresamente a "Trabajo Social" (incluye prácticas de intervención profesional del Trabajo Social); "Servicios Sociales", "Política Social" y "Métodos y Técnicas de investigación en trabajo social". u De otro, un conjunto de Ciencias Sociales 
Aplicadas, entre las que figuran: "Sociología", "Antropología", "Psicología”, "Derecho" y "Economía”.

El hecho de que en la propuesta curricular y en el perfil profesional quedan bien diferenciados los títulos de Grado de Educación Social y Trabajo Social, como es deseable y esperable en función de los fundamentos epistemológicos y profesionales que definen a una y otra formación académica, nada impide -al igual que podrá suceder con otros ámbitos formativos y profesionalesque deban buscarse puntos de convergencia entre ambos; posiblemente ya en la formación que habiliten uno y otro en las Universidades, a través de materias que puedan tener contenidos que compartan sus respectiva áreas de conocimiento. Aunque también, y sobre todo, en la realización de Postgrados en los que la formación que se diseñe no sólo aconseje sino que incluso exija -por el bien de la ciudadanía, y no sólo de las instituciones y de los profesionales de uno y otro ámbito- que se haga coparticipadamente.

Asumimos, en esta línea, la necesidad de que la Educación Social por sí misma y en función de las propuestas que puedan formularse a través de su confluencia con otras titulaciones y profesiones educativas y sociales, promueva el diseño y la realización de actividades de postgrado, de carácter interdepartamental e interuniversitario, tal y como aparece contemplado en la legislación hasta ahora vigente. En principio, y con un horizonte temporal de corto-medio plazo, contando con la exigencia de que la responsabilidad de organizar estos programas corresponde a las universidades, y buscando que efectivamente se pueda favorecer la "especialización del estudiante en su formación académica, profesional o investigadora" (a través de las enseñanzas conducentes a la obtención de los títulos de Master o Doctor), consideramos prioritario la cre- ación y realización de un Programa de postgrado en Pedagogía Social, en el que puedan participar distintas universidades favoreciendo la movilidad de los estudiantes y del profesorado que participe en su desarrollo.

El futuro dirá, con los plazos que se estimen convenientes, en qué medida podrán ser adaptados, diseñados e implementados otros programas de postgrado, a partir de los ya existentes o en función de la implantación de los nuevos Grados en Educación Social y Pedagogía, que creemos deberán ser congruentes con las realidades y necesidades de cada Comunidad Autónoma, de sus Universidades, Facultades y Departamentos... en un contexto europeo y mundial precisado de las mejores transiciones posibles entre lo local y lo global. Que las "menciones", “ámbitos” o "perfiles profesionales" de cada título constituyan el soporte de la oferta que se diseñe parece, en principio, lo deseable.

La colaboración entre las universidades y el mundo profesional, más en concreto entre los Centros con títulos de Educación y los Colegios Profesionales de Educadores y Educadoras Sociales, también forma parte de las necesidades inherentes a la mejor planificación y desarrollo de los nuevos procesos formativos, en los Grados y Postgrados, así como en el establecimiento de las vías más idóneas para la inserción profesional y laboral de los titulados.

El camino recorrido, con abundantes realizaciones conjuntas, y los desafíos del futuro obligan a que el entendimiento y la cooperación sean dos pilares básicos para que la Convergencia Europea de la Educación Superior haga que la Educación Social sea un ejemplo claro de que es factible alcanzar -como se apuntaba en la Declaración de Bolonia- un desarrollo armónico de los procesos formativos y de las oportunidades de los estudiantes respecto del mercado laboral y de sus condiciones de ciudadanía, f avoreciendo el aprendizaje a lo largo de toda la vida. Un propósito para el que la Pedagogía 
Social, además de acreditar un sugerente pasado, está en condiciones de aportar nuevas y estimables orientaciones de futuro.

\section{Referencias bibliográficas}

Aldecoa, F. (2004): “¿Qué añade la Constitución para Europa a la construcción europea?" en Documentación Social, 134, pp. 11-26.

ANECA (2005a): Libro Blanco de Grado en Pedagogía y Educación Social, vols. 1 y 2. Madrid: Agencia Nacional de Evaluación de la Calidad y Acreditación-ANECA.

ANECA (2005b): Libro Blanco del Título de Grado en Trabajo Social. Madrid: Agencia Nacional de Evaluación de la Calidad y Acre ditación-ANECA.

Caride, J. A. (2005): Las fronteras de la Pedagogía Social: perspectivas científica e histórica. Barcelona: Gedisa.

Castells, M. (1997): La era de la información: economía, sociedad y cultura, vol. I. Madrid: Alianza Editorial.

Comisión Europea (2000): La Política Social y de empleo en Europa: una política para el ciudadano. Luxemburgo: Oficina de Publicaciones Oficiales de las Comunidades Europeas.

Delors, J. (coord., 1996): La educación encierra un tesoro. Madrid: Santillana-UNESCO,

Del Río, S. (2004): "Convención, sociedad civil y Constitución europea: la Unión”. Documentación Social, 134, pp. 27-40.

Fontaine, P. (2003): Doce lecciones sobre Europa. Luxemburgo: Oficina de Publicaciones Oficiales de las Comunidades Europeas.
Giner, S. (2000): "Cultura republicana y política del porvenir". En Giner, S. (comp.): $L a$ cultura de la democracia. Barcelona: Ariel, pp. 137-172.

Giner, S. (2005): “Ciudadanía pública y sociedad civil republicana”. Documentación Social, 139, pp. 13-34.

Quintanilla, M. A. (1995): “Nuevas ideas para la Universidad”. Revista de Educación, 308, pp. 131-140.

March, M. (1998): "El educador social: una figura profesional surgida de diversas prácticas e identidades profesionales". En FEAPES: Actas del I Congreso Estatal del Educador Social: presente y futuro en la Educación Social. Barcelona: FEAPES-Biblária, pp. 49-67.

Martínez, M. y Esteban, F. (2005): “Una propuesta de formación ciudadana para el EEES". Revista Española de Pedagogía, 230, pp. $63-84$.

M ayor Zaragoza, M. (1998): "La Univer sidad del siglo XXI en la perspectiva de la UNESCO". En Porta, J. y Lladonosa, M. (coords.): La Universidad en el cambio de siglo. Madrid: Alianza Editorial, pp. 309320.

Morin, E. (1998): "Sobre la reforma de la Universidad". En Porta, J. y Lladonosa, M. (coords.): La Universidad en el cambio de siglo. Madrid: Alianza Editorial, pp. 19-28.

Muñoz Sedano, A. (ed., 1994): El educador social: profesión y formación universitaria. Madrid: Popular.

Ortega, J. (1999): “Educación Social Especializada, concepto y profesión”. En Ortega, J. (coord.): Educación Social Especializada. Barcelona: Ariel, pp. 13-41.

[ 30 ] SIPS - PEDAGOGÍA SOCIAL (ISSN-1139-1723) № 14 TERCERA ÉPOCA 
Pérez Serrano, G. (2003): Pedagogía SocialEducación Social: construcción científica e intervención práctica. Madrid: Narcea.

Planella, J. y Vilar, J. (coords., 2006): La Peda gogía Social en la sociedad de la información. Barcelona: Editorial UOC.

Sáez, J. (2003): “Cambio de rumbo en la construcción de la Pedagogía Social. Revisión y propuestas". Pedagogía Social. Revista Interuniversitaria, 10 (segunda época), pp. 27-59.

Sáez, J. (2006): "La reorganización conceptual del campo de conocimiento de la Pedagogía Social: hacia la construcción de la Pedagogía Social y de la Educación Social". En Planella, J. y Vilar, J. (coords.): La Peda gogía Social en la sociedad de la información. Barcelona: Editorial UOC, pp. 17-59.

Sáez, J. y García Molina, J. (2006): Pedagogía Social: pensar la Educación Social como profesión. Madrid: Alianza Editorial.
Vilar, J. (2006): “Comenzando una nueva etapa: Retos de futuro para el ejercicio responsable de la educación social y la pedagogía social". En Planella, J. y Vilar, J. (coords.): La Pedago gía Social en la sociedad de la información. Barcelona: Editorial UOC, pp. 163-195.

DireCCIÓN DEL AUTOR: José Antonio Caride Gómez. Universidad de Santiago de Compostela. Facultad de Ciencias de la Educación. Campus Sur. 15782 Santiago de Compostela.

Correo electrónico: hecaride@usc.es

Fecha de recepción del artículo: 29.X.2006

Fecha de aceptación definitiva: 14.XII.2007 